\title{
Positive Regulation of Cytolytic Process
}

National Cancer Institute

\section{Source}

National Cancer Institute. Positive Regulation of Cytolytic Process. NCI Thesaurus. Code C40724.

Any process that increases the frequency, rate or extent of cytolysis. 